\title{
Diffractograms of semicrystalline polymers
}

\author{
Yvonne P. Mascarenhas \\ Sao Carlos Institute of Physics \\ Institute of Advanced Studies at Sao Carlos \\ University of Sao Paulo
}

Polyaniline (PANI) and derivatives received considerable attention due to their interesting properties as polymeric organic electricity conductors and technological applications. In this work, using XRD, SAXS and electron microscopy, we present some structural results regarding different synthesis conditions and their influence on the corresponding electrical conductivity. 